\title{
Identification of the association between HMMR expression and progression of hepatocellular carcinoma via construction of a co-expression network
}

\author{
DONGLAN LU ${ }^{1 *}$, XUE BAI $^{1 *}$, QIYUAN ZOU ${ }^{2}$, ZUHUAN GAN $^{3}$ and YUFENG LV ${ }^{3}$ \\ ${ }^{1}$ Department of Medical Oncology, The Second Affiliated Hospital of Guilin Medical University, Guilin, \\ Guangxi Zhuang Autonomous Region 541199; Departments of ${ }^{2}$ Medicine 1 and ${ }^{3}$ Oncology, Affiliated Langdong Hospital of \\ Guangxi Medical University, Nanning, Guangxi Zhuang Autonomous Region 530021, P.R. China
}

Received January 17, 2019; Accepted January 4, 2020

DOI: $10.3892 / \mathrm{ol} .2020 .11844$

\begin{abstract}
The aim of the present study was to identify key genes involved in the progression of hepatocellular carcinoma (HCC). According to the theory of the multistep process of hepatocarcinogenesis and weighted gene co-expression network analysis, hub genes associated with the progression of HCC were identified using the gene expression profiles of patients with normal to chronic hepatitis/cirrhosis and dysplastic nodules to HCC. An independent dataset was used to verify the association between hub gene and clinical phenotype. The diagnostic and prognostic value of hub genes regarding HCC were evaluated. Gene set enrichment analysis (GSEA) was performed to explore the function of hub genes. A co-expression gene module positively associated with HCC progression was identified. Combined with a protein-protein interaction (PPI) network, a total of 10 common hub genes common to both the module of interest and the PPI network were selected as hub genes. Hyaluronan mediated motility receptor (HMMR) was selected as the candidate gene and was significantly upregulated in HCC at the mRNA and protein expression levels. HMMR is a promising diagnostic biomarker for $\mathrm{HCC}$, and is also associated with its progression. The expression of HMMR was positively correlated with HCC tumor grade, pathological stage, tumor stage and Ishak score. The expression of HMMR was an independent prognostic factor compared with clinicopathological
\end{abstract}

Correspondence to: Dr Yufeng Lv, Department of Oncology, Affiliated Langdong Hospital of Guangxi Medical University, 60 Xiangbin Road, Nanning, Guangxi Zhuang Autonomous Region 530021, P.R. China

E-mail: tougaozhuanyong_lv@126.com

\section{${ }^{*}$ Contributed equally}

Key words: hepatocellular carcinoma, weighted gene co-expression network analysis, HMMR, multistep process of hepatocarcinogenesis features. Patients with high expression levels of HMMR exhibited a less favorable prognosis. GSEA identified 6 representative gene sets that were associated with cancer. Overall, HMMR may serve an important role in HCC and may have potential as a biomarker of HCC diagnosis and progression.

\section{Introduction}

Liver cancer, of which $75-85 \%$ of cases consist of hepatocellular carcinoma (HCC), was the sixth most commonly diagnosed cancer and the fourth leading cause of cancer-associated death worldwide in 2018 (1). HCC is one of the few types of cancer which has had a continued increase in incidence over the last decade (2). The risk factors for HCC include chronic infection with hepatitis C or hepatitis B virus, high alcohol intake, aflatoxin B1, obesity, smoking and type 2 diabetes (3-5). Hepatocarcinogenesis is considered to be a multistep process evolving from normal to chronic hepatitis/cirrhosis and dysplastic nodules to HCC $(6,7)$. Curative treatment options are limited to surgical resection of the tumor or liver transplantation; however, $>70 \%$ of patients with HCC will encounter recurrence within 5 years after surgery (8-10). The specific mechanisms underlying the progression from healthy liver to chronic hepatitis/cirrhosis and dysplastic nodules to HCC are still elusive. The investigation of these mechanisms may help identify potential therapeutic targets to prevent the development and recurrence of HCC and biomarkers of these processes may help clinicians monitor this disease progression.

Previously, with the development of high-throughput and microarray technology, gene expression profiles have been used to identify genes associated with the progression of HCC (11-13). However, the majority of these studies focused on the screening of differentially expressed genes without considering the correlations between genes, despite the fact that genes with similar expression patterns may be functionally related (14). Weighted gene co-expression network analysis (WGCNA) can be used to analyze the associations between gene sets and indicators of tumor progression, including tumor stages and grades $(15,16)$. In the present 
study, the various stages of progression from healthy liver to chronic hepatitis/cirrhosis and dysplastic nodules to HCC were treated as phenotypes. It was hypothesized that the expression patterns of certain genes would be closely associated with these phenotypes.

According to the theory of the multistep process of hepatocarcinogenesis $(6,7)$ and WGCNA, the present study aimed to identify network-centric genes associated with HCC progression by constructing a co-expression network using gene expression profiles from normal to chronic hepatitis/cirrhosis and dysplastic nodules to HCC. Hyaluronan mediated motility receptor (HMMR) was identified to exhibit a strong correlation with the progression of HCC and may represent a promising marker for the prevention and treatment of HCC.

\section{Materials and methods}

Data collection. The microarray dataset (GSE89377) of HCC was retrieved from the Gene Expression Omnibus (GEO) database (ncbi.nlm.nih.gov/geo/) using the R (http://www.R-project.org/) package GEOquery (17) in RStudio (http://www.rstudio.com/). The GSE89377 dataset was collected using Illumina HumanHT-12 version 3.0 expression beadchip (Illumina, Inc.) and was used to construct co-expression networks and identify hub genes in the present study. There were 13 healthy liver tissue samples and 94 tissues covering 9 stages of HCC progression in the GSE89377 dataset, including low grade chronic hepatitis $(n=8)$, high grade chronic hepatitis $(n=12)$, cirrhosis $(n=12)$, low grade dysplastic nodules $(n=11)$, high grade dysplastic nodules $(n=11)$, early HCC $(n=5)$, HCC with grade $1(n=9)$, HCC with grade $2(n=12)$ and HCC with grade $3(n=14)$. The gene expression profiles had been normalized using quantile normalization with GenPlex version 3.0 by Jung Woo Eun from The Catholic University of Korea. An independent dataset including RNA-sequencing data and clinical information was obtained from The Cancer Genome Atlas (TCGA) database (cancer.gov/tcga) to further verify the association of hub genes and clinical phenotypes. The gene expression profiles of GSE87630 (18) based on the GPL6947 dataset included $64 \mathrm{HCC}$ and 30 non-tumor profiles used to validate the aberrant expression of the hub genes. The gene expression profiles of GSE87630 were processed using the lumi package (19) in R. As these data are publicly available and open-access, ethical approval was not necessary for the present study.

Weighted gene co-expression network construction. Probes were filtered by variance as recommended (15), and the 4,881 probes with the highest variance were selected from 48,803 probes. The 'WGCNA' package (15) was used to construct a co-expression network for the 4,881 probes according to the protocols of WGCNA and R software. First, Pearson's correlation matrices were performed for all pair-wise genes. Subsequently, an adjacency matrix was constructed using a power adjacency function $\left[\alpha_{\mathrm{mn}}=\operatorname{Power}\left(\mathrm{S}_{\mathrm{mn}} \beta\right)=\left|\mathrm{S}_{\mathrm{mn}}\right| \beta ; \alpha_{\mathrm{mn}}\right.$, adjacency between two genes; $S_{\mathrm{mn}}$, Pearson's correlations between two genes]. $\beta$ is a soft-thresholding parameter that emphasizes strong correlations between genes and penalizes weak correlations. In the present study, the power of $\beta=9$ (scale-free
$\left.\mathrm{R}^{2}=0.85\right)$ was chosen in accordance with the scale-free topology criterion (Fig. 1A). Next, the adjacency was transformed into a topological overlap matrix that measured the network connectivity of a gene, defined as the sum of its adjacency with all other genes for network generation (20). Finally, the 'cutreeStaticColor' function was applied to classify similar expression genes into gene modules (minModuleSize=30; mergeCutHeight=0.25).

Identification of clinically significant modules and functional enrichment analysis. Gene significance (GS) was defined as the $\log _{10}$ transformation of the P-value in the linear regression between gene expression and HCC progress. Module significance (MS) was the mean GS for all the genes in a module. In general, the module with the absolute MS ranked first or second (ranked by MS) amongst all modules was considered as the module correlating with HCC progression. In the present study, the module exhibiting the strongest positive correlation with HCC progression was selected for further analysis and termed the primary module. Module eigengenes (MEs) were considered as the major components in the principal component analysis for each gene module and the expression patterns of all genes could be summarized into a single characteristic expression profile within a given module. The correlation between MEs and clinical traits was calculated to identify the relevant module. As there is more potential of oncogene as a marker or therapeutic target (21), the focus was on modules that are positively associated with HCC progression. In addition, functional Gene Ontology (GO) enrichment analysis and Kyoto Encyclopedia of Genes and Genomes (KEGG) pathway analysis were performed using the 'clusterProfiler' R package (22) in order to uncover the biologic function of genes in the primary module. P-value [adjusted by false discovery rate $(\mathrm{FDR})]<0.01$ was set as the cutoff criteria.

Identification of hub genes. In the present study, hub genes in the primary module were defined by module connectivity, measured by the absolute value of the Pearson's correlation (cor.geneModuleMembership $\geq 0.8$ ) and clinical trait relationship, measured by absolute value of the Pearson's correlation (cor.geneTraitSignificance $\geq 0.7$ ) (23). Furthermore, a protein-protein interaction (PPI) network was constructed by uploading all genes in the primary module to the Search Tool for the Retrieval of Interacting Genes (STRING) database (24). Overall, 50 genes with the highest connectivity degree were defined as hub genes in the PPI network. The connectivity degree for each gene in the PPI network was calculated using the 'cytoHubba' (25) plugin in Cytoscape version 3.6.1 software (26). The hub genes common to both co-expression network and PPI networks were selected as 'real' hub genes and included for further analyses.

Hub gene validation and survival analysis. The Human Protein Atlas (27) was used to validate the expression of the hub genes at the protein level. The Human Protein Atlas is a publicly available database, all data and images are available for free download and non-commercial use. For validation of the correlation of hub genes and HCC progression, $371 \mathrm{HCC}$ 


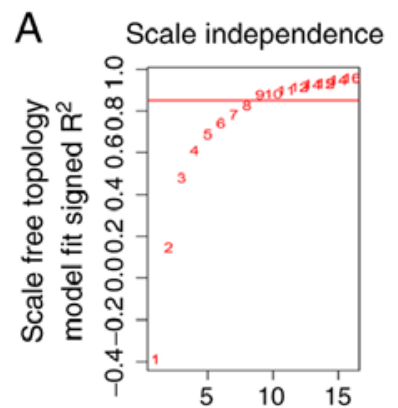

Soft threshold (power)

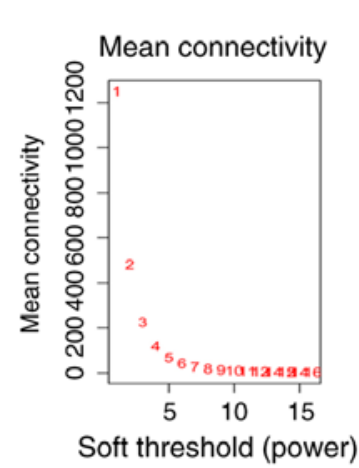

C

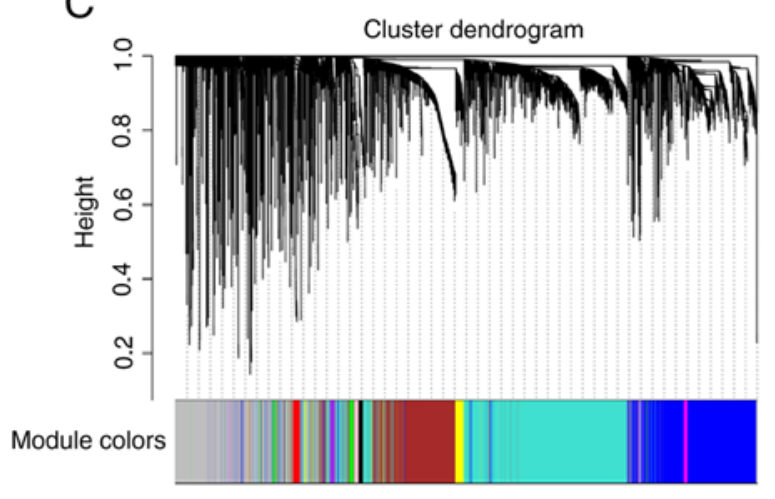

B

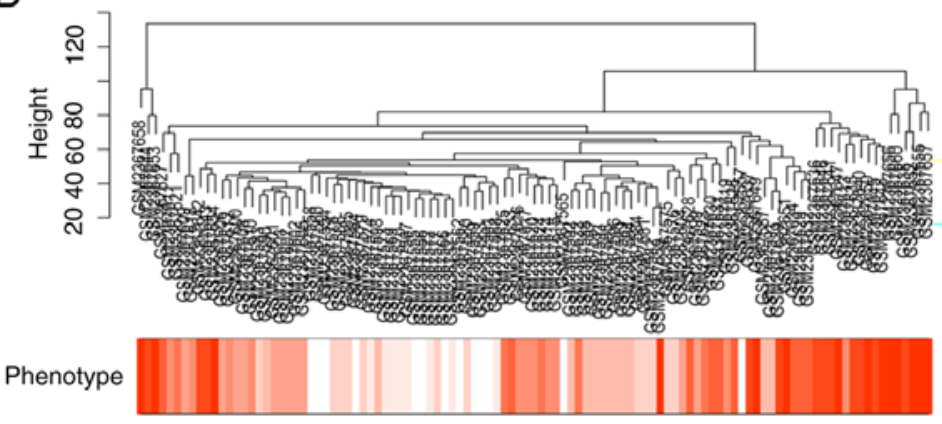

D

Sample dendrogram and trait heatmap

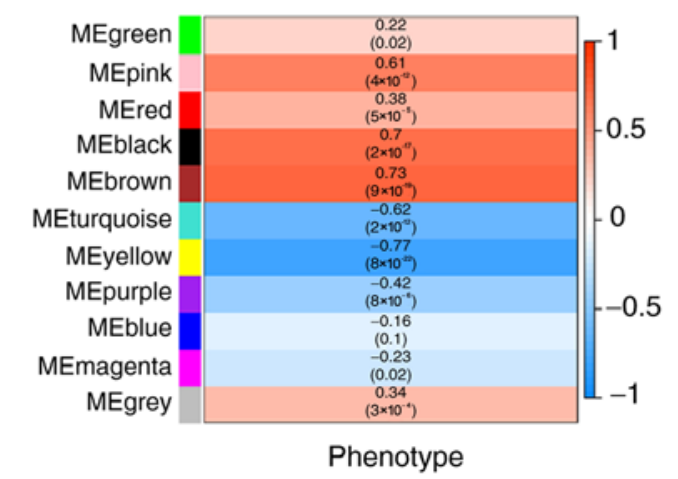

Figure 1. Weighted correlation network analysis in GSE89377. (A) Analysis of the scale-free fit index for various soft-thresholding powers ( $\beta$ ). (B) Clustering dendrogram of 107 samples and the clinical traits. Color intensity is proportional to higher grade (red) based on the theory of the multistep process hepatocarcinogenesis. (C) Dendrogram of genes clustered based on a dissimilarity measure (1-Topological Overlap Matrix). (D) Heatmap of the correlation between module eigengenes and clinical phenotypes in the GSE89377 dataset.

samples from TCGA database were analyzed to calculate the Pearson's correlation coefficient between hub gene expression and certain clinicopathological features. To evaluate the impact of the hub genes on the prognosis of patients with HCC, overall and disease-free survival rate were analyzed using Gene Expression Profiling Interactive Analysis tools (GEPIA) (28). The predictive value for prognosis between hub genes and routine clinicopathological factors were compared using univariate and multivariate Cox regression analyses in an HCC dataset from TCGA (TCGA-LIHC). These clinicopathological factors comprised alpha feto protein (AFP) (29), vascular invasion (30), Ishak score (32), and tumor pathological staging (33). $\mathrm{P}<0.05$ were set as the cut-off criteria for significance.

Gene set enrichment analysis (GSEA). 371 HCC samples from RNA-sequencing data (displayed as read counts) were divided into two groups (high vs. low) according to the expression level of the candidate gene and the median expression value was selected as the cut-off point. The RNA-sequencing data was normalized using the limma package (34) in R. To determine the potential function of candidate gene, GSEA (35) was performed between the 2 groups. Hallmark gene sets summarize and represent specific well-defined biological states or processes and display coherent expression. These gene sets were generated by a computational methodology based on identifying overlaps between gene sets in other MSigDB collections (36) and retaining genes that display coordinate expression. Thus, the Hallmark gene sets (37) were selected as the reference gene sets. FDR $<0.05$ was set as the cut-off criteria.

Statistical analysis. The expression levels of the hub genes were analyzed using unpaired Student's t-tests in the comparison of two groups. ANOVA and Dunnett's post-hoc test were used for multiple comparisons using the multcomp package (CRAN.R-project.org/package=multcomp) in R. Univariate/multivariate Cox proportional hazards analyses and Kaplan-Meier survival analysis with log-rank method were used to compare survival between the two groups of patients. $\mathrm{P}<0.05$ was considered to indicate a statistically significant difference.

\section{Results}

Weighted co-expression network construction and primary module identification. Overall, 13 healthy liver samples and 94 samples from different stages (low grade chronic hepatitis, high grade chronic hepatitis, cirrhosis, low grade dysplastic nodules, high grade dysplastic nodules, early HCC, HCC with grade $1, \mathrm{HCC}$ with grade 2 and $\mathrm{HCC}$ with grade 3 ) of $\mathrm{HCC}$ progression were included in co-expression analysis (Fig. 1B). In the present study, the power of $\beta=9$ (scale free $R^{2}=0.85$ ) was selected as the soft-threshold to ensure a scale-free network (Fig. 1A) and 11 modules were identified (Fig. 1C). The highest association between module and phenotype was revealed to be between the yellow module and clinical phenotype $(r=-0.77$; $\mathrm{P}=8 \times 10^{-22}$; Fig. 1D); however, the brown module and clinical 

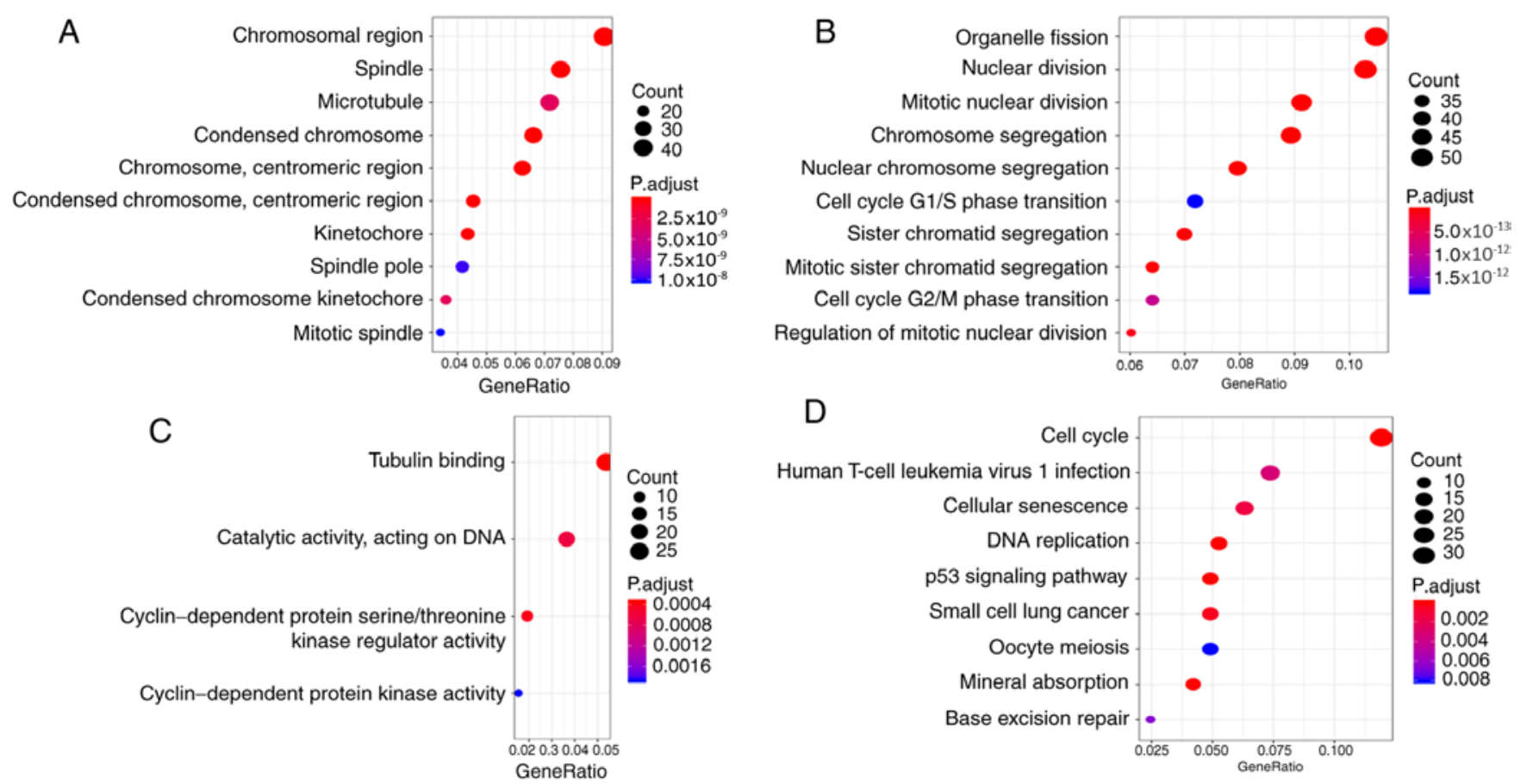

Figure 2. Significantly enriched Gene Ontology annotations and enriched KEGG and Genomes pathways of genes in the brown module. (A) Cellular component. (B) Biological process. (C) Molecular function. (D) KEGG pathway analysis. KEGG, Kyoto Encyclopedia of Genes.

phenotype exhibited the highest positive correlation $(\mathrm{r}=0.73$; $\mathrm{P}=9 \times 10^{-19}$; Fig. 1D). This indicated that the brown module genes may be oncogenes and the yellow module genes may be tumor suppressor genes. As there is more potential of oncogene as a marker or therapeutic target (21), the brown module was selected and included in subsequent analyses.

To explore the biological relevance of the brown module, GO and KEGG enrichment analyses were performed for 671 genes using the 'clusterProfiler' package. GO enrichment analyses contained three parts: Cellular component (CC; Fig. 2A); biological process (BP; Fig. 2B); and molecular function (MF; Fig. 2C). The brown module genes were involved in mitotic-related CCs and BPs, such as 'microtubule', 'nuclear division' and 'cell cycle G2/M phase transition'. While the brown module genes were involved in kinase-related MFs, such as 'cyclin-dependent protein kinase activity'. The results of enrichment analyses indicated that the brown module genes were involved in various cancer-associated pathways (Fig. 2D), such as 'cell cycle' and 'p53 signaling pathway'. The brown module genes associated with HCC are also involved in some viral infection-related pathways, such as human T-cell leukemia virus 1 infection.

Identification of hub genes. In the present study, 20 genes with high connectivity (cor.geneModuleMembership; $\geq 0.8$ ) and high clinical trait relationship (cor.geneTraitSignificance; $\geq 0.7$ ) in the brown module were selected as hub genes in WGCNA. A PPI network (Table SI; Fig. 3) was constructed using Cytoscape according to the STRING database and 50 genes with the highest connectivity degree were defined as hub genes in the PPI network. Then, a total of 10 genes (TOP2A, CDC20, CCNB2, PRC1, UBE2C, PTTG1, KIF20A, HMMR,
NUSAP1 and RACGAP1) common to both the co-expression network and the PPI network were selected as 'real' hub genes (Table I). The aberrant expression data of the 10 hub genes were validated in an independent data set (Fig. 4). HMMR was selected as the candidate gene for further analysis due to the few existing reports about its role in HCC.

Hub gene validation and survival analysis. The expression of HMMR at the mRNA and protein levels were both significantly higher in HCC tissue compared with healthy liver tissue (Fig. 5A-C). In the GSE89377 dataset, HMMR exhibited diagnostic efficiency for HCC with an area under curve (AUC) $=0.949$, sensitivity $=0.875$ and specificity $=0.910$ (Fig. 5D). Based the results of WGCNA, the expression of HMMR was positively correlated with the progression of HCC (cor.geneTraitSignificance $\left.\mathrm{r}=0.706 ; \mathrm{P}=2.00 \times 10^{-17}\right)$. This correlation was validated in the HCC dataset from TCGA ( $\mathrm{r}=0.290 ; \mathrm{P}=3.57 \times 10^{-6}$; Fig. 5E). The expression of HMMR was positively correlated with HCC pathological stage $(r=0.062 ; \mathrm{P}=0.008)$, tumor $(\mathrm{T})$ stage $\left(\mathrm{r}=0.069 ; \mathrm{P}=4.19 \times 10^{-4}\right)$ and Ishak score (Pearson correlation coefficient $=0.178 ; \mathrm{P}=0.004 ;$ Fig. $5 \mathrm{E}$ ). Using GEPIA tools, it was revealed that patients with higher expression levels of HMMR exhibited significantly shorter overall survival (Fig. 5F, left) and disease-free survival rate (Fig. 5F, right). Furthermore, the expression of HMMR is an independent prognostic factor compared with routine clinicopathological features, not only in overall survival but also disease-free survival rate, in the HCC dataset from TCGA (Tables II and III) (multivariate Cox regression analysis $\mathrm{P}<0.05$ ).

GSEA. To analyze the function of HMMR in HCC, GSEA was conducted to compare HMMR with hallmark gene sets. 371 HCC samples were divided into two groups (high vs. 
Table I. Common hub genes in the brown module from weighted gene co-expression network analysis in GSE89377 and PPI network.

\begin{tabular}{llccc}
\hline & & \multicolumn{2}{c}{ Weighted gene co-expression network analysis } & PPI network \\
\cline { 3 - 5 } Probe & Gene & cor.geneModuleMembership & cor.geneTraitSignificance & Connectivity degree \\
\hline ILMN_1686097 & TOP2A & 0.956 & 0.748 & 142 \\
ILMN_1663390 & CDC20 & 0.961 & 0.724 & 121 \\
ILMN_1801939 & CCNB2 & 0.962 & 0.712 & 111 \\
ILMN_1728934 & PRC1 & 0.962 & 0.725 & 102 \\
ILMN_1714730 & UBE2C & 0.954 & 0.710 & 97 \\
ILMN_1753196 & PTTG1 & 0.946 & 0.706 & 92 \\
ILMN_1695658 & KIF20A & 0.938 & 0.715 & 92 \\
ILMN_1781942 & HMMR & 0.916 & 0.706 & 97 \\
ILMN_1726720 & NUSAP1 & 0.933 & 0.709 & 86 \\
ILMN_2077550 & RACGAP1 & 0.959 & 0.717 & 83
\end{tabular}

PPI, Protein-Protein Interaction.

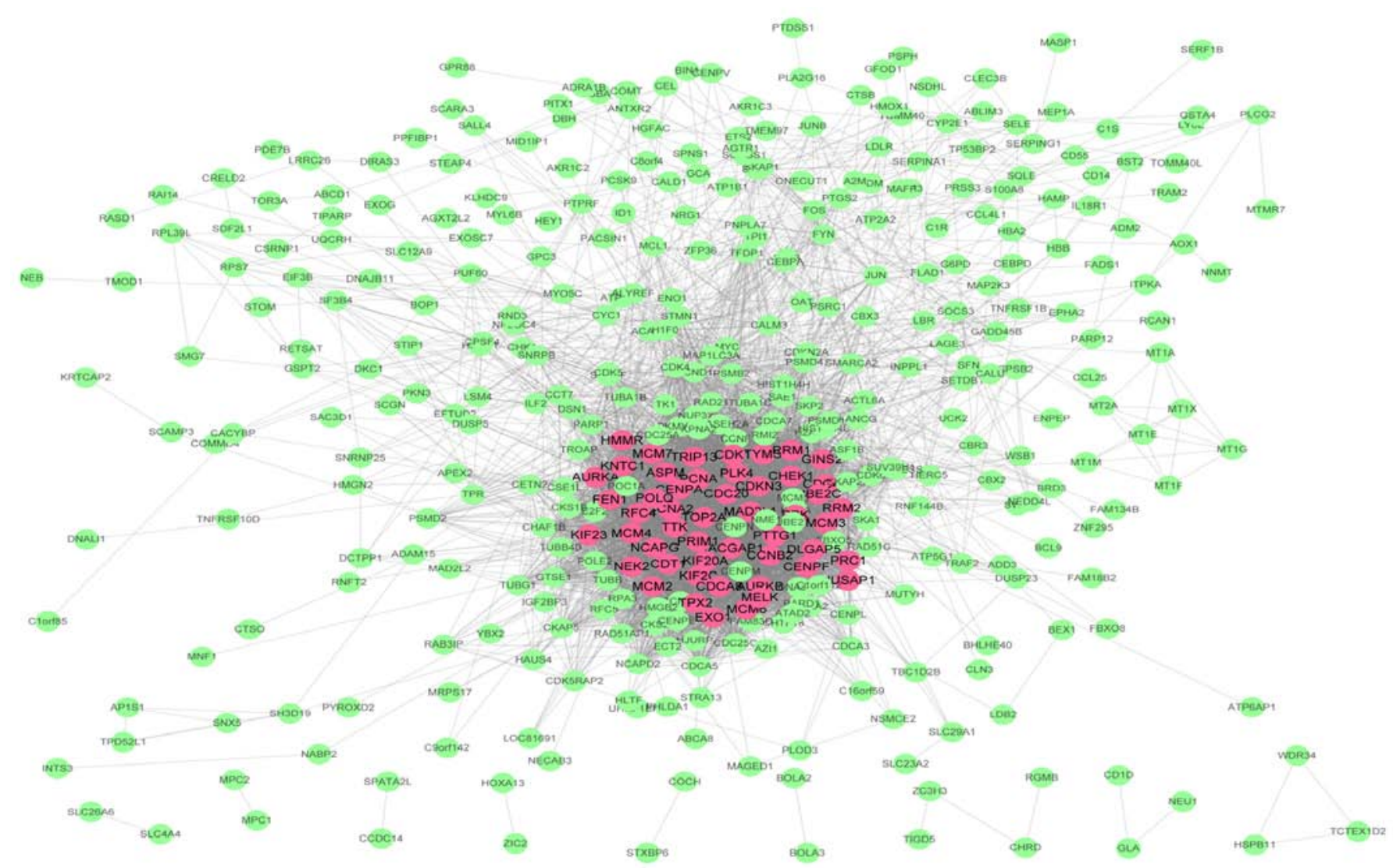

Figure 3. Protein-protein interaction networks of genes in the brown module. Red nodes were 50 genes with the highest connectivity degree and grey edges represent the interaction between proteins.

low) according to the HMMR median expression level (2.62). Under the cut-off criteria of FDR $<0.05$, a total of 8 functional gene sets were enriched. Overall, 6 representative gene sets were significantly associated with cancer, including 'mitotic spindle', ' $\mathrm{G}_{2} / \mathrm{M}$ checkpoint', 'MYC targets v1', 'E2F targets', 'DNA repair' and 'mTORC1 signaling' (Fig. 6).

\section{Discussion}

In previous years, the concept of multi-step human hepatocarcinogenesis has been well documented $(6,7,38)$. Chronic liver inflammation can result in repeated cell injury, death and regeneration cycles, resulting in subsequent epigenetic 
Expression of the ten hub genes in GSE87630
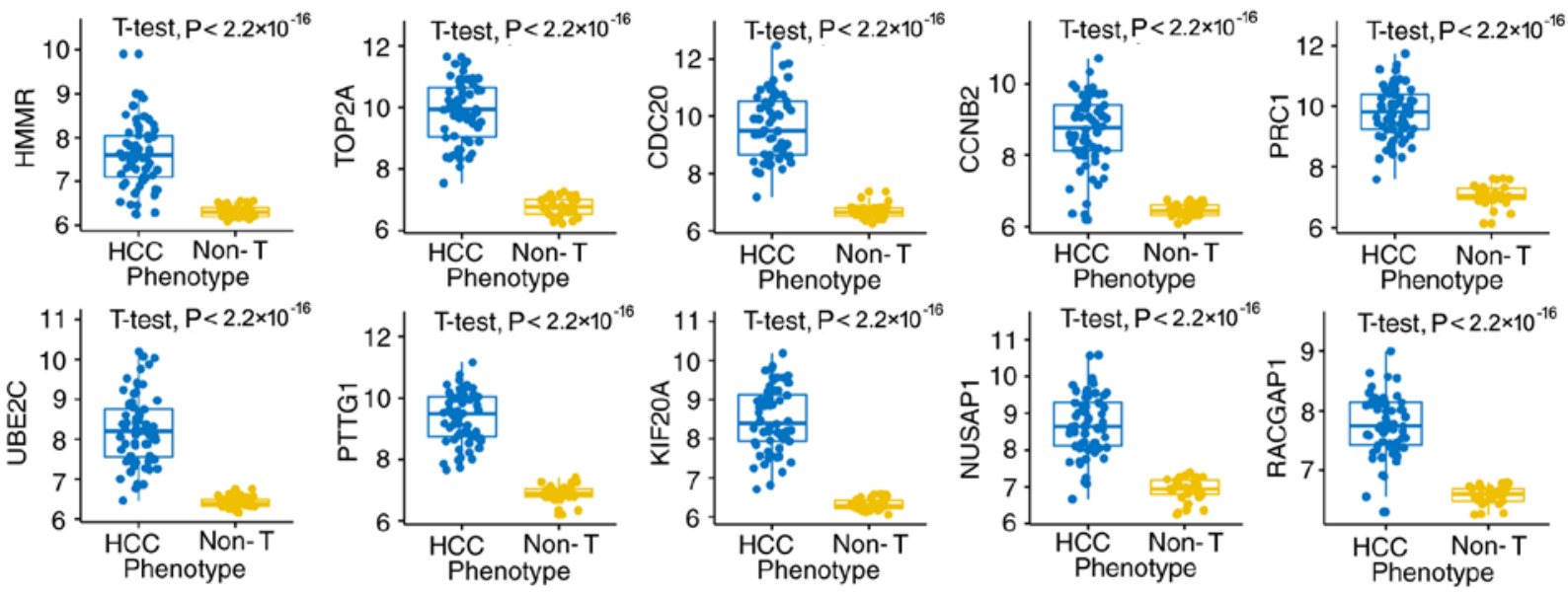

Figure 4. Aberrant expression of hub genes validated in HCC and non-tumor phenotypes of the GSE87630 dataset. HCC, hepatocellular carcinoma. Non-T, non-tumor.
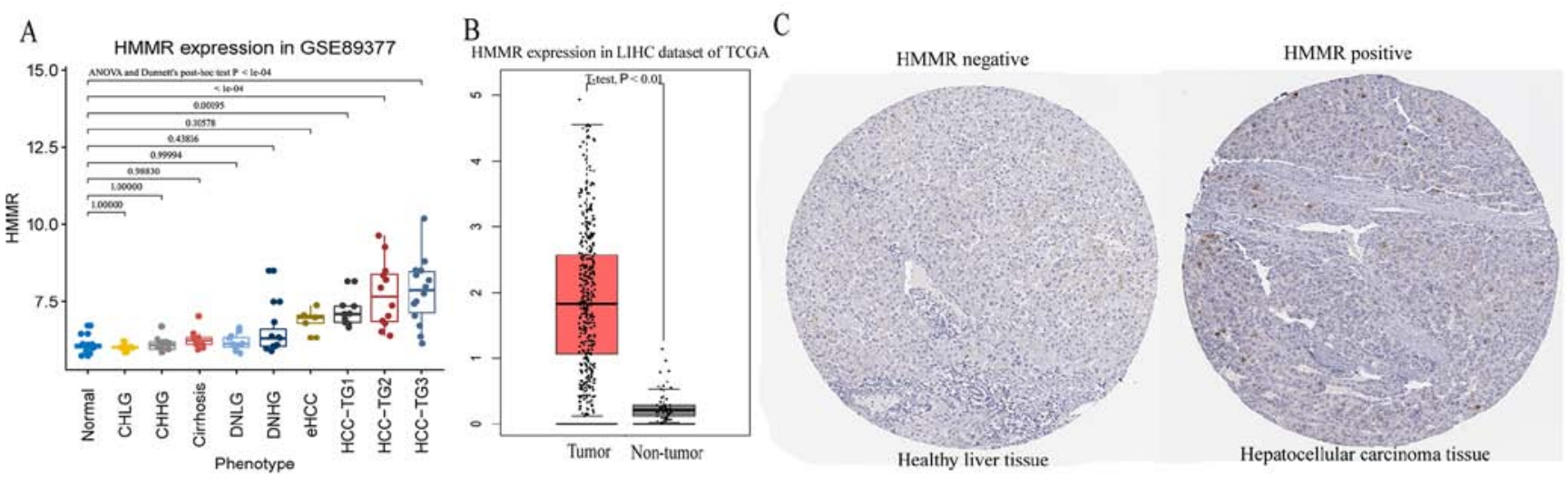

D

$\mathrm{E}$

$\mathrm{F}$
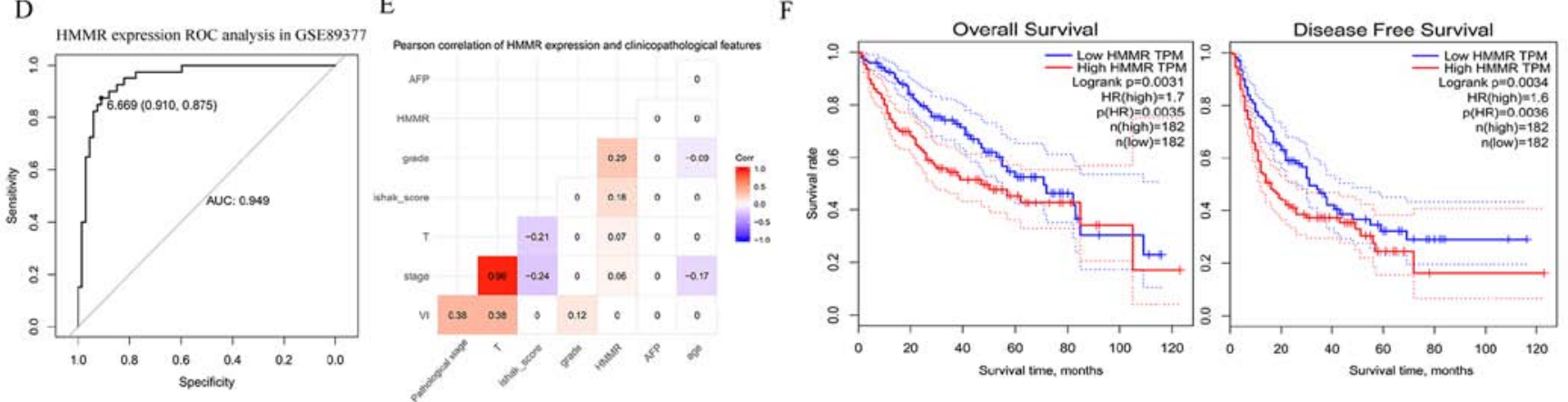

Figure 5. Validation of aberrant expression of HMMR at transcription and protein levels and the prognostic value of HMMR in HCC. (A) Expression of HMMR at different pathological stages from normal to chronic hepatitis/cirrhosis and dysplastic nodules to HCC in the GSE89377 dataset. (B) Expression of HMMR between tumor tissues and non-tumor tissues in hepatocellular carcinoma dataset from TCGA. (C) HMMR protein was upregulated in hepatocellular carcinoma (images.proteinatlas.org/2433/6865_B_8_6.jpg) compared with healthy liver tissue (images.proteinatlas.org/2433/6892_A_8_4.jpg) (antibody CAB002433) using data from the Human Protein Atlas database. The healthy liver tissue was from a female (patient ID: 1846) and the hepatocellular carcinoma tissue was from a male (patient ID: 2325). (D) ROC curves of the expression of HMMR for hepatocellular carcinoma diagnosis in the GSE89377 dataset. (E) Pearson correlation between HMMR expression and routine clinicopathological features. This shows the correlation coefficient when P<0.01. (F) Kaplan-Meier curves obtained using the median value of HMMR expression to separate patients into high- and low-expression groups in Gene Expression Profiling Interactive Analysis. HCC, hepatocellular carcinoma; HMRR, Hyaluronan mediated motility receptor; LIHC, liver hepatocellular carcinoma; TCGA, The Cancer Genome Atlas; ROC, receiver operating characteristic; HR, hazard ratio; T, tumor; CHLG, chronic hepatitis with low grade; CHHG, chronic hepatitis with high grade; DNLG, dysplastic nodules with low grade; DNHG, dysplastic nodules with high grade; eHCC, early hepatocellular carcinoma; HCC-TG1, hepatocellular carcinoma with grade 1; HCC-TG2, hepatocellular carcinoma with grade 2; HCC-TG3, hepatocellular carcinoma with grade 3.

and genetic alterations of hepatocytes (39). Phenotypically abnormal precursor hepatic lesions, including cirrhotic nodules, low-grade dysplastic nodules and high-grade dysplastic nodules dedifferentiate and gradually evolve to HCC (40). This process exists on a biologic continuum and may occur simultaneously at various rates throughout 
Table II. Univariate and multivariate COX regression analyses for overall survival in hepatocellular carcinoma dataset of The Cancer Genome Atlas.

\begin{tabular}{|c|c|c|c|c|c|c|}
\hline \multirow[b]{2}{*}{ Factors } & \multicolumn{3}{|c|}{ Univariate analysis } & \multicolumn{3}{|c|}{ Multivariate analysis } \\
\hline & P-value & HR & $\mathrm{HR}, 95 \% \mathrm{CI}$ & P-value & HR & $\mathrm{HR}, 95 \% \mathrm{CI}$ \\
\hline Sex (male vs. female) & 0.262 & 1.225 & $0.859-1.745$ & - & - & - \\
\hline Age, years $(>65$ vs. $\leq 65)$ & 0.186 & 1.265 & $0.893-1.791$ & - & - & - \\
\hline AFP, ng/ml (>20 vs. $\leq 20)(29)$ & $0.026^{\mathrm{a}}$ & 1.641 & $1.061-2.540$ & 0.563 & 1.150 & $0.716-1.846$ \\
\hline Vascular invasion (positive vs. negative) (30) & 0.155 & 1.351 & $0.892-2.047$ & - & - & - \\
\hline Child-pugh score (B/C vs. A) (31) & 0.184 & 1.614 & $0.796-3.270$ & - & - & - \\
\hline Ishak score (5-6 vs. 0-4) (32) & 0.497 & 0.829 & $0.483-1.424$ & - & - & - \\
\hline Tumor Grade (G3/4 vs. G1/2) & 0.542 & 1.119 & $0.780-1.604$ & - & - & - \\
\hline Pathological T stage (T3/4 vs. T1/2) (33) & $<0.001^{\mathrm{a}}$ & 2.537 & $1.783-3.609$ & 0.889 & 1.153 & $0.155-8.607$ \\
\hline Pathological stage (III/IV vs. I/II) (33) & $<0.001^{\mathrm{a}}$ & 2.446 & $1.687-3.545$ & 0.634 & 1.620 & $0.222-11.808$ \\
\hline HMMR expression level (high vs. low) & $<0.001^{\mathrm{a}}$ & 2.136 & $1.498-3.044$ & $0.007^{\mathrm{a}}$ & 1.917 & $1.192-3.085$ \\
\hline
\end{tabular}

${ }^{\text {a }}<0.05$. T, tumor; HR, hazard ratio; CI, confidence interval; HMMR, hyaluronan mediated motility receptor; AFP, alpha-fetoprotein .

Table III. Univariate and multivariate Cox regression analyses for disease-free survival in HCC dataset of TCGA.

\begin{tabular}{|c|c|c|c|c|c|c|}
\hline \multirow[b]{2}{*}{ Clinicopathological factors } & \multicolumn{3}{|c|}{ Univariate analysis } & \multicolumn{3}{|c|}{ Multivariate analysis } \\
\hline & P-value & HR & $\mathrm{HR}(95 \% \mathrm{CI})$ & P-value & HR & $\mathrm{HR}(95 \% \mathrm{CI})$ \\
\hline Sex (male vs. female) & 0.919 & 1.019 & $0.714-1.454$ & & & \\
\hline Age, years (>65 vs. $\leq 65)$ & 0.081 & 1.043 & $0.739-1.472$ & & & \\
\hline AFP, ng/ml (>20 vs. $\leq 20)(29)$ & 0.496 & 1.149 & $0.771-1.712$ & & & \\
\hline Vascular invasion (positive vs. negative) (30) & $0.029^{\mathrm{a}}$ & 1.540 & $1.045-2.268$ & 0.397 & 1.198 & $0.788-1.821$ \\
\hline Child-pugh score (B/C vs. A) (31) & 0.246 & 1.542 & $0.742-3.204$ & & & \\
\hline Ishak score (5-6 vs. 0-4) (32) & 0.623 & 1.116 & $0.721-1.727$ & & & \\
\hline Tumor Grade (G3/4 vs. G1/2) & 0.829 & 1.039 & $0.733-1.474$ & & & \\
\hline Pathological T stage (T3/4 vs. T1/2) (33) & $<0.001^{\mathrm{b}}$ & 2.940 & $2.071-4.173$ & 0.518 & 0.512 & $0.067-3.902$ \\
\hline Pathological stage (III/IV vs. I/II) (34) & $<0.001^{\mathrm{b}}$ & 2.885 & $2.009-4.142$ & 0.126 & 4.769 & $0.644-35.330$ \\
\hline HMMR expression level (high vs. low) & $0.002^{\mathrm{b}}$ & 1.697 & $1.213-2.373$ & $0.038^{\mathrm{a}}$ & 1.527 & $1.024-2.277$ \\
\hline
\end{tabular}

${ }^{\mathrm{a}} \mathrm{P}<0.05,{ }^{\mathrm{b}} \mathrm{P}<0.01$. HCC, hepatocellular carcinoma; TCGA, The Cancer Genome Atlas; HR, hazard ratio; CI, confidence interval; T, tumor; G, grade; AFP, alpha-fetoprotein; HMMR, Hyaluronan mediated motility receptor expression.

the liver; however, the specific molecular mechanisms underlying this process are yet to be elucidated. In the present study, several modules associated with this process were identified using WGCNA. In particular, genes in the brown module exhibited a strong positive correlation with this process, indicating that gene expression in the module gradually increase as the process progresses. Functional enrichment analysis revealed that genes in the brown module significantly influenced cell cycle-associated biological processes, for example 'cell cycle $\mathrm{G}_{2} / \mathrm{M}$ phase transition', 'cell cycle $\mathrm{G}_{1} / \mathrm{S}$ phase transition' and 'mitotic nuclear division', and cancer-related pathways, including 'p53 signaling pathway' and 'cell cycle'.

Markers which accurately reflect the process from normal to chronic hepatitis/cirrhosis and dysplastic nodules to HCC are lacking in clinical practice and novel candidate molecules are needed. In the present study, according to the theory of the multistep process of hepatocarcinogenesis and WGCNA, a total of 10 hub genes common to the primary module and PPI network were selected as hub genes, including TOP2A, CDC20, CCNB2, PRC1, UBE2C, PTTG1, KIF20A, HMMR, NUSAP1 and RACGAP1. Previous studies had reported almost all ten genes to be associated with the progression of HCC (41-49). HMMR was chosen as the candidate gene, since few studies have identified its role in HCC (50). HMMR (also known as CD168/IHABP/RHAMM) (51) is highly expressed in various solid tumors and it is described as a cancer-associated antigen, which is involved in both tumorigenesis and progression/metastasis (52-56). HMMR was identified as a 

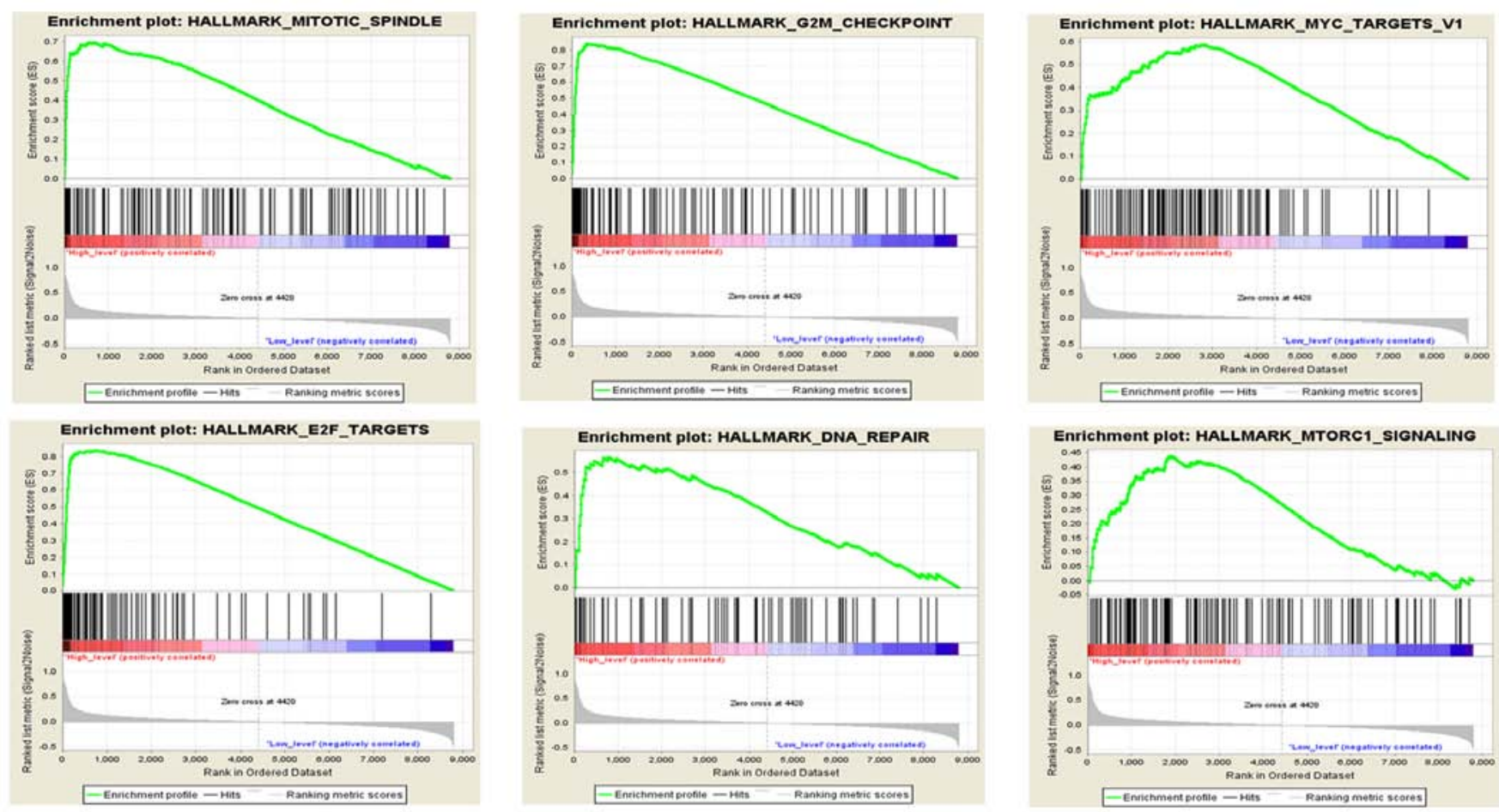

Figure 6. Gene set enrichment analysis. Overall, 6 representative gene sets were significantly enriched in hepatocellular carcinoma samples with high Hyaluronan mediated motility receptor expression.

breast cancer susceptibility gene (57) and was once considered an ideal target antigen for immunotherapy of acute myeloid leukemia (58); however, the association between HMMR and HCC has been rarely reported.

In the present study, HMMR was significantly upregulated in HCC tissue compared with healthy liver tissue at both the mRNA and protein expression levels. HMMR is a promising diagnostic biomarker for $\mathrm{HCC}(\mathrm{AUC}=0.949$; sensitivity $=0.875$; specificity $=0.910$ ). In addition, the progression of $\mathrm{HCC}$ was associated with the upregulation of HMMR. Notably, the expression of HMMR was positively correlated with HCC tumor grade, pathological stage, T stage and Ishak score. Patients with $\mathrm{HCC}$ with higher expression levels of HMMR exhibited significantly shorter overall survival and disease-free survival times. Moreover, the expression of HMMR remained an independent prognostic factor compared with routine clinicopathological features. The current results indicated that HMMR may serve as a biomarker of HCC progression. Thus, patients with HCC and high expression levels of HMMR have a higher risk for recurrence and should be followed up more frequently than the routine schedule.

In order to reveal the function of HMMR in HCC, GSEA was performed. Overall, 6 representative gene sets, including 'mitotic spindle', ' $\mathrm{G}_{2} / \mathrm{M}$ checkpoint', 'MYC targets v1', 'E2F targets', 'DNA repair' and 'mTORC1 signaling', were significantly associated with cancer and enriched in samples with high expression levels of HMMR. This indicates that HMMR may interact with these genes or pathways to promote the progression of HCC. The present findings may improve our understanding of the role of HMMR in HCC and inform future research.
Notably, there were certain limitations to the present study. Firstly, the expression of HMMR was quantified and the values may vary on different platforms. The establishment of a standard is required before being applied to clinical practice. Secondly, as the present study only performed a bioinformatic analysis, including GSEA analysis to help identify the function of HMMR in HCC, it is not clear whether HMMR expression is causal or merely a biomarker of HCC progression. Whether HMMR can be used as a therapeutic target for HCC requires further molecular experimental verification.

In conclusion, the present study revealed that patients with HCC with high expression of HMMR exhibit a less favorable prognosis, suggesting that HMMR may serve an important role in HCC and has potential as a biomarker of HCC diagnosis and progression.

\section{Acknowledgements}

Not applicable.

\section{Funding}

This research was supported by the Youth Science Foundation of Guangxi Medical University, Nanning, China (grant no. GXMUYSF 201716).

\section{Availability of data and materials}

The dataset of GSE89377 and GSE87630 are available from the Gene Expression Omnibus (https://www.ncbi.nlm.nih. gov/geo). The dataset of TCGA-LIHC are available from The Cancer Genome Atlas (cancer.gov/tcga). 


\section{Authors' contributions}

YL designed the study and reviewed the manuscript. DL and $\mathrm{XB}$ analyzed the data and wrote the manuscript. ZG and QZ assisted with analyzing the data and writing the manuscript. All authors read and approved the final manuscript.

\section{Ethics approval and consent to participate}

Not applicable.

\section{Patient consent for publication}

Not applicable.

\section{Competing interests}

The authors declare that they have no competing interests.

\section{References}

1. Bray F, Ferlay J, Soerjomataram I, Siegel RL, Torre LA and Jemal A: Global cancer statistics 2018: GLOBOCAN estimates of incidence and mortality worldwide for 36 cancers in 185 countries. CA Cancer J Clin 68: 394-424, 2018.

2. Forner A, Reig M and Bruix J: Hepatocellular carcinoma. Lancet 391: 1301-1314, 2018.

3. Heidelbaugh JJ and Bruderly M: Cirrhosis and chronic liver failure: Part I. Diagnosis and evaluation. Am Fam Physician 74: 756-762, 2006.

4. Alter MJ: Epidemiology of hepatitis C virus infection. World J Gastroenterol 13: 2436-2441, 2007.

5. El-Serag HB, Hampel $\mathrm{H}$ and Javadi F: The association between diabetes and hepatocellular carcinoma: A systematic review of epidemiologic evidence. Clin Gastroenterol Hepatol 4: 369-380, 2006.

6. Marquardt JU, Seo D, Andersen JB, Gillen MC, Kim MS, Conner EA, Galle PR, Factor VM, Park YN and Thorgeirsson SS: Sequential transcriptome analysis of human liver cancer indicates late stage acquisition of malignant traits. J Hepatol 60: 346-353, 2014.

7. Kudo M: Multistep human hepatocarcinogenesis: Correlation of imaging with pathology. J Gastroenterol 44 (Suppl 19): S112-S118, 2009

8. Rahbari NN, Mehrabi A, Mollberg NM, Müller SA, Koch M, Büchler MW and Weitz J: Hepatocellular carcinoma: Current management and perspectives for the future. Ann Surg 253 453-469, 2011

9. Hasegawa K, Kokudo N, Makuuchi M, Izumi N, Ichida T, Kudo M, Ku Y, Sakamoto M, Nakashima O, Matsui O and Matsuyama Y: Comparison of resection and ablation for hepatocellular carcinoma: A cohort study based on a Japanese nationwide survey. J Hepatol 58: 724-729, 2013.

10. Poon RT, Fan ST, Lo CM, Liu CL and Wong J: Long-term survival and pattern of recurrence after resection of small hepatocellular carcinoma in patients with preserved liver function: Implications for a strategy of salvage transplantation. Ann Surg 235: 373-382, 2002.

11. Cheng J, Wei D, Ji Y, Chen L, Yang L, Li G, Wu L, Hou T, Xie L, Ding $\mathrm{G}$, et al: Integrative analysis of DNA methylation and gene expression reveals hepatocellular carcinoma-specific diagnostic biomarkers. Genome Med 10: 42, 2018.

12. Kalinich M, Bhan I, Kwan TT, Miyamoto DT, Javaid S, LiCausi JA, Milner JD, Hong X, Goyal L, Sil S, et al: An RNA-based signature enables high specificity detection of circulating tumor cells in hepatocellular carcinoma. Proc Natl Acad Sci USA 114: 1123-1128, 2017.

13. SiaD,Jiao Y,Martinez-Quetglas I,Kuchuk O, Villacorta-Martin C, Castro de Moura M, Putra J, Camprecios G, Bassaganyas L, Akers $\mathrm{N}$, et al: Identification of an immune-specific class of hepatocellular carcinoma, based on molecular features. Gastroenterology 153: 812-826, 2017.

14. Tavazoie S, Hughes JD, Campbell MJ, Cho RJ and Church GM: Systematic determination of genetic network architecture. Nat Genet 22: 281-285, 1999.
15. Langfelder $P$ and Horvath S: WGCNA: An R package for weighted correlation network analysis. BMC Bioinformatics 9: 559, 2008.

16. Chen L, Yuan L, Qian K, Qian G, Zhu Y, Wu CL, Dan HC, Xiao Y and Wang $\mathrm{X}$ : Identification of biomarkers associated with pathological stage and prognosis of clear cell renal cell carcinoma by co-expression network analysis. Front Physiol 9: 399, 2018.

17. Davis S and Meltzer PS: GEOquery: A bridge between the gene expression omnibus (GEO) and BioConductor. Bioinformatics 23: 1846-1847, 2007.

18. Woo HG, Choi JH, Yoon S, Jee BA, Cho EJ, Lee JH, Yu SJ, Yoon $\mathrm{JH}$, Yi NJ, Lee KW, et al: Integrative analysis of genomic and epigenomic regulation of the transcriptome in liver cancer. Nat Commun 8: 839, 2017.

19. Du P, Kibbe WA and Lin SM: Lumi: A pipeline for processing Illumina microarray. Bioinformatics 24: 1547-1548, 2008.

20. Yip AM and Horvath S: Gene network interconnectedness and the generalized topological overlap measure. BMC Bioinformatics 8: $22,2007$.

21.

22. Yu G, Wang LG, Han Y and He QY: clusterProfiler: An R package for comparing biological themes among gene clusters. OMICS 16: 284-287, 2012.

23. Horvath S and Dong J: Geometric interpretation of gene coexpression network analysis. PLoS Comput Biol 4: e1000117, 2008.

24. Szklarczyk D, Gable AL, Lyon D, Junge A, Wyder S, Huerta-Cepas J, Simonovic M, Doncheva NT, Morris JH, Bork P, et al: STRING v11: Protein-protein association networks with increased coverage, supporting functional discovery in genome-wide experimental datasets. Nucleic Acids Res 47 (D1): D607-D613, 2019.

25. Chin $\mathrm{CH}$, Chen SH, Wu HH, Ho CW, Ko MT and Lin CY: cytoHubba: Identifying hub objects and sub-networks from complex interactome. BMC Syst Biol 8 (Suppl 4): S11, 2014.

26. Shannon P, Markiel A, Ozier O, Baliga NS, Wang JT, Ramage D, Amin N, Schwikowski B and Ideker T: Cytoscape: A software environment for integrated models of biomolecular interaction networks. Genome Res 13: 2498-2504, 2003.

27. Uhlen M, Fagerberg L, Hallstrom BM, Lindskog C, Oksvold P, Mardinoglu A, Sivertsson Å, Kampf C, Sjöstedt E, Asplund A, et al: Proteomics. Tissue-based map of the human proteome. Science 347: 1260419, 2015.

28. Tang Z, Li C, Kang B, Gao G, Li C and Zhang Z: GEPIA: A web server for cancer and normal gene expression profiling and interactive analyses. Nucleic Acids Res 45 (W1): W98-W102, 2017.

29. Nomura F, Ohnishi K and Tanabe Y: Clinical features and prognosis of hepatocellular carcinoma with reference to serum alpha-fetoprotein levels. Analysis of 606 patients. Cancer 64: 1700-1707, 1989.

30. Llovet JM, Schwartz M and Mazzaferro V: Resection and liver transplantation for hepatocellular carcinoma. Semin Liver Dis 25: 181-200, 2005.

31. Cholongitas E, Papatheodoridis GV, Vangeli M, Terreni N, Patch D and Burroughs AK: Systematic review: The model for end-stage liver disease-should it replace Child-Pugh's classification for assessing prognosis in cirrhosis? Aliment Pharmacol Ther 22: 1079-1089, 2005.

32. Ishak K, Baptista A, Bianchi L, Callea F, De Groote J, Gudat F, Denk H, Desmet V, Korb G, MacSween RN, et al: Histological grading and staging of chronic hepatitis. J Hepatol 22: 696-699, 1995.

33. Sobin LH, Gospodarowicz MK and Wittekind C (eds): TNM classification of malignant tumours. 7th edition. John Wiley \& Sons, 2009.

34. Ritchie ME, Phipson B, Wu D, Hu Y, Law CW, Shi W and Smyth GK: Limma powers differential expression analyses for RNA-sequencing and microarray studies. Nucleic Acids Res 43: e47, 2015 .

35. Mootha VK, Lindgren CM, Eriksson KF, Subramanian A, Sihag S, Lehar J, Puigserver P, Carlsson E, Ridderstråle M, Laurila E, et al: PGC-1alpha-responsive genes involved in oxidative phosphorylation are coordinately downregulated in human diabetes. Nat Genet 34: 267-273, 2003.

36. Subramanian A, Tamayo P, Mootha VK, Mukherjee S, Ebert BL, Gillette MA, Paulovich A, Pomeroy SL, Golub TR, Lander ES and Mesirov JP: Gene set enrichment analysis: A knowledge-based approach for interpreting genome-wide expression profiles. Proc Natl Acad Sci USA 102: 15545-15550, 2005.

37. Liberzon A, Birger C, Thorvaldsdóttir H, Ghandi M, Mesirov JP and Tamayo P: The molecular signatures database (MSigDB) hallmark gene set collection. Cell Syst 1: 417-425, 2015. 
38. Niu ZS, Niu XJ, Wang WH and Zhao J: Latest developments in precancerous lesions of hepatocellular carcinoma. World J Gastroenterol 22: 3305-3314, 2016.

39. Batts KP and Ludwig J: Chronic hepatitis. An update on terminology and reporting. Am J Surg Pathol 19: 1409-1417, 1995.

40. Choi JY, Lee JM and Sirlin CB: CT and MR imaging diagnosis and staging of hepatocellular carcinoma: Part I. Development, growth, and spread: Key pathologic and imaging aspects. Radiology 272 : 635-654, 2014.

41. Wong N, Yeo W, Wong WL, Wong NL, Chan KY, Mo FK, Koh J, Chan SL, Chan AT, Lai PB, et al: TOP2A overexpression in hepatocellular carcinoma correlates with early age onset, shorter patients survival and chemoresistance. Int J Cancer 124: 644-652, 2009.

42. Li J, Gao JZ, Du JL, Huang ZX and Wei LX: Increased CDC20 expression is associated with development and progression of hepatocellular carcinoma. Int J Oncol 45: 1547-1555, 2014.

43. Gao CL, Wang GW, Yang GQ, Yang $\mathrm{H}$ and Zhuang L: Karyopherin subunit- $\alpha 2$ expression accelerates cell cycle progression by upregulating CCNB2 and CDK1 in hepatocellular carcinoma. Oncol Lett 15: 2815-2820, 2018.

44. Chen J, Rajasekaran M, Xia H, Zhang X, Kong SN, Sekar K, Seshachalam VP, Deivasigamani A, Goh BK, Ooi LL, et al: The microtubule-associated protein PRC1 promotes early recurrence of hepatocellular carcinoma in association with the Wnt/ $\beta$-catenin signalling pathway. Gut 65: 1522-1534, 2016.

45. Ieta K, Ojima E, Tanaka F, Nakamura Y, Haraguchi N, Mimori K, Inoue $\mathrm{H}$, Kuwano $\mathrm{H}$ and Mori $\mathrm{M}$ : Identification of overexpressed genes in hepatocellular carcinoma, with special reference to ubiquitin-conjugating enzyme E2C gene expression. Int J Cancer 121: 33-38, 2007.

46. Molina-Jimenez F, Benedicto I, Murata M, Martín-Vílchez S, Seki T, Antonio Pintor-Toro J, Tortolero M, Moreno-Otero R, Okazaki K, Koike $\mathrm{K}$, et al: Expression of pituitary tumor-transforming gene 1 (PTTG1)/securin in hepatitis B virus (HBV)-associated liver diseases: Evidence for an HBV $X$ protein-mediated inhibition of PTTG1 ubiquitination and degradation. Hepatology 51: 777-787, 2010.

47. Lu M, Huang X, Chen Y, Fu Y, Xu C, Xiang W, Li C, Zhang S and Yu C: Aberrant KIF20A expression might independently predict poor overall survival and recurrence-free survival of hepatocellular carcinoma. IUBMB Life 70: 328-335, 2018.

48. Zhang M, Yang D, Liu X, Liu Y, Liang J, He H, Zhong K, Lin L, Tao G, Zhang C and Zhou J: Expression of Nusapl in the surgical margins of hepatocellular carcinoma and its association with early recurrence. Nan Fang Yi Ke Da Xue Xue Bao 33: 937-938, inside back cover, 2013 (In Chinese).

49. Yang XM, Cao XY, He P, Li J, Feng MX, Zhang YL, Zhang XL, Wang YH, Yang Q, Zhu L, et al: Overexpression of Rac GTPase activating protein 1 contributes to proliferation of cancer cells by reducing hippo signaling to promote cytokinesis. Gastroenterology 155: 1233-1249 e22, 2018.
50. Wurmbach E, Chen YB, Khitrov G, Zhang W, Roayaie S, Schwartz M, Fiel I, Thung S, Mazzaferro V, Bruix J, et al: Genome-wide molecular profiles of $\mathrm{HCV}$-induced dysplasia and hepatocellular carcinoma. Hepatology 45: 938-947, 2007.

51. Willemen Y, Van den Bergh JM, Bonte SM, Anguille S, Heirman C, Stein BM, Goossens H, Kerre T, Thielemans K, Peeters M, et al: The tumor-associated antigen RHAMM (HMMR/CD168) is expressed by monocyte-derived dendritic cells and presented to T cells. Oncotarget 7: 73960-73970, 2016.

52. Rein DT, Roehrig K, Schondorf T, Lazar A, Fleisch M, Niederacher D, Bender HG and Dall P: Expression of the hyaluronan receptor RHAMM in endometrial carcinomas suggests a role in tumour progression and metastasis. J Cancer Res Clin Oncol 129: 161-164, 2003.

53. Kalmyrzaev B, Pharoah PD, Easton DF, Ponder BA and Dunning AM; SEARCH Team: Hyaluronan-mediated motility receptor gene single nucleotide polymorphisms and risk of breast cancer. Cancer Epidemiol Biomarkers Prev 17: 3618-3620, 2008.

54. Buttermore ST, Hoffman MS, Kumar A, Champeaux A, Nicosia SV and Kruk PA: Increased RHAMM expression relates to ovarian cancer progression. J Ovarian Res 10: 66, 2017.

55. Koelzer VH, Huber B, Mele V, Iezzi G, Trippel M, Karamitopoulou E, Zlobec I and Lugli A: Expression of the hyaluronan-mediated motility receptor RHAMM in tumor budding cells identifies aggressive colorectal cancers. Hum Pathol 46: 1573-1581, 2015.

56. Ishigami S, Ueno S, Nishizono $\mathrm{Y}$, Matsumoto $\mathrm{M}$, Kurahara $\mathrm{H}$, Arigami T, Uchikado Y, Setoyama T, Arima H, Yoshiaki K, et al: Prognostic impact of CD168 expression in gastric cancer. BMC Cancer 11: 106, 2011.

57. Pujana MA, Han JD, Starita LM, Stevens KN, Tewari M, Ahn JS, Rennert G, Moreno V, Kirchhoff T, Gold B, et al: Network modeling links breast cancer susceptibility and centrosome dysfunction. Nat Genet 39: 1338-1349, 2007.

58. Snauwaert S, Vanhee S, Goetgeluk G, Verstichel G, Van Caeneghem Y, Velghe I, Philippé J, Berneman ZN, Plum J, Taghon T, et al: RHAMM/HMMR (CD168) is not an ideal target antigen for immunotherapy of acute myeloid leukemia. Haematologica 97: 1539-1547, 2012.

This work is licensed under a Creative Commons Attribution-NonCommercial-NoDerivatives 4.0 International (CC BY-NC-ND 4.0) License. 